\title{
Value tradeoffs propel and inhibit behavior: Validating the 19 refined values in four countries
}

\author{
Shalom H. Schwartz* ${ }^{*}$, Jan Cieciuch $¥, \S$, Michele Vecchioneף, Claudio Torres\#, \\ Ozlem Dirilen-Gumus\| \& Tania Butenko† \\ * Department of Psychology, The Hebrew University of Jerusalem, Jerusalem, Israel \\ † International Laboratory of Socio-cultural Research, National Research University Higher School of Economics, Moscow, Russian \\ Federation \\ ¥ Cardinal Stefan Wyszynski University, Warsaw, Poland \\ $\S$ University of Zurich, Zurich, Switzerland \\ I Department of Psychology, "Sapienza" University of Rome, Rome, Italy \\ \# Department of Psychology, Universidade de Brasília, Brasilia, Brazil \\ || Department of Psychology, Hacettepe University, Ankara, Turkey
}

\section{Correspondence}

Shalom H. Schwartz, Department of Psychology, The Hebrew University of Jerusalem, Jerusalem, 91905, Israel. E-mail:msshasch@mscc.huji.ac.il

Received: 4 October 2015

Accepted: 5 June 2016

http://dx.doi.org/10.1002/ejsp.2228

Correction added on 31 July 2017, after first online publication: a note was missing from Figure 2 and has been corrected in this version of the article.

\begin{abstract}
We assess the predictive and discriminant validity of the basic values in the refined Schwartz value theory by examining how value tradeoffs predict behavior in Italy, Poland, Russia, and the USA. One thousand eight hundred and fifty-seven respondents reported their values and rated their own and a partner's behavior. Multigroup confirmatory factor analysis supported the distinctiveness of the 19 values and the 19 self-rated and other-rated behaviors. Multidimensional scaling analyses supported the circular motivational order of the 19 values. Findings affirmed the theorizing that behavior depends upon tradeoffs between values that propel and values that inhibit it. Across four countries, value importance, behavior frequency, and gender failed to moderate the strength of value-behavior relations. This raises the question of the conditions under which the widely cited assumption that normative pressure weakens value-behavior relations holds.
\end{abstract}

How many basic human values is it worthwhile for researchers to distinguish? By "values", we refer to "desirable transsituational goals, varying in importance, that serve as guiding principles in the life of a person or other social entity" (Schwartz, 1994: p. 21). The recently refined theory of basic values (Schwartz et al., 2012) suggests that it is sometimes desirable to distinguish 19 motivationally distinct values. Sixteen of these values are more narrowly defined than the 10 values in the widely used original theory (Schwartz, 1992), and three remain the same. The refined theory retains the key assumption that values form a circular motivational continuum. It argues, however, that partitioning this continuum into a finer set of meaningful values can yield increased heuristic and predictive power. Moreover, the refined theory identifies the expected patterns of association between these values and any external variable by specifying the order of the 19 values around the motivational circle. The 19 values are arrayed on the same two basic dimensions as in the original theory.

In order to establish the benefit of adopting the refined theory, it is necessary first to establish that individuals across cultures actually do discriminate the 19 values. Beyond that, it is necessary to demonstrate that each of these values relates in distinctive ways to other variables such as attitudes, behaviors and demographic variables. Schwartz et al. (2012) assessed the discriminant validity of the refined values in a study in 10 countries, using a newly developed values instrument. They also examined the order of the values around the circle and their associations with numerous attitude, belief, and background variables. They found substantial support for the discriminant validity of the refined theory, some for the predictive validity and, with some exceptions noted later, evidence for the circular order of the values. Table 1 presents the 19 values of the refined theory.

Critically, however, there is only minimal evidence that the 19 narrowly defined values predict behavior distinctively. This is critical because a major reason for studying values is the assumption that they can explain, influence, and predict behavior (Roccas \& Sagiv, 2010; Rokeach, 1973). A recent study related the 19 values to behavior in a Russian sample (Schwartz \& Butenko, 2014). In that study, both values and behavior were based only on self-reports, however. Thus, shared method variance and recall of relevant past behavior when reporting own values may have accounted for or inflated the observed value-behavior correlations. 
Table 1. The 19 values in the refined theory, each defined in terms of its motivational goal

\begin{tabular}{|c|c|}
\hline Value & $\begin{array}{l}\text { Conceptual definitions in terms of } \\
\text { motivational goals }\end{array}$ \\
\hline Self-direction thought & $\begin{array}{l}\text { Freedom to cultivate one's own ideas and } \\
\text { abilities }\end{array}$ \\
\hline Self-direction action & Freedom to determine one's own actions \\
\hline Stimulation & Excitement, novelty, and change \\
\hline Hedonism & Pleasure and sensuous gratification \\
\hline Achievement & Success according to social standards \\
\hline Power-dominance & Power through exercising control over people \\
\hline Power-resources & $\begin{array}{l}\text { Power through control of material and social } \\
\text { resources }\end{array}$ \\
\hline Face & $\begin{array}{l}\text { Maintaining one's public image and avoiding } \\
\text { humiliation }\end{array}$ \\
\hline Security-personal & Safety in one's immediate environment \\
\hline Security-societal & Safety and stability in the wider society \\
\hline Tradition & $\begin{array}{l}\text { Maintaining and preserving cultural, family } \\
\text { or religious traditions }\end{array}$ \\
\hline Conformity-rules & $\begin{array}{l}\text { Compliance with rules, laws, and formal } \\
\text { obligations }\end{array}$ \\
\hline $\begin{array}{l}\text { Conformity- } \\
\text { interpersonal }\end{array}$ & $\begin{array}{l}\text { Avoidance of upsetting or harming other } \\
\text { people }\end{array}$ \\
\hline Humility & $\begin{array}{l}\text { Recognizing one's insignificance in the larger } \\
\text { scheme of things }\end{array}$ \\
\hline Universalism-nature & Preservation of the natural environment \\
\hline Universalism-concern & $\begin{array}{l}\text { Commitment to equality, justice and } \\
\text { protection for all people }\end{array}$ \\
\hline Universalism-tolerance & $\begin{array}{l}\text { Acceptance and understanding of those who } \\
\text { are different from oneself }\end{array}$ \\
\hline Benevolence caring & Devotion to the welfare of in-group members \\
\hline $\begin{array}{l}\text { Benevolence- } \\
\text { dependability }\end{array}$ & $\begin{array}{l}\text { Being a reliable and trustworthy member of } \\
\text { the in-group }\end{array}$ \\
\hline
\end{tabular}

From Schwartz et al. (2012).

The current research examines relations of values to behavior using both other-rated and self-rated behavior. Moreover, we gathered data in four socio-economically and culturally diverse countries, Italy, Poland, Russia, and the USA. We assess whether each of 19 values exhibits its expected pattern of associations with behaviors, whether these associations are present in each sample and whether gender and normative pressure moderate them. Earlier research has demonstrated associations of the 10 values in the original theory with a wide variety of behaviors. Examples include helping behavior (Lönnqvist, Walkowitz, Wichardt, Lindeman, \& Verkasalo, 2009), creative behavior (Kasof, Chen, Himsel, \& Greenberger, 2007), interpersonal violence (Knafo, Daniel, \& Khoury-Kassabri, 2008), risky sexual behavior (Goodwin et al., 2002), environmental behavior (Schultz \& Zelezny, 1998), drug use (Tamayo, Nicaretta, Ribeiro, \& de Barbosa, 1995), internet use (Hartman, Shim, Barber, \& O'Brien, 2006), choice of medical specialty (Eliason \& Schubot, 1995), consumer choices (Doran, 2009), and musical preferences (Gardikiotis \& Blatzis, 2012). Neither these nor other studies provide evidence for the predictive validity of the refined values nor do they explicitly examine the effects on behavior of tradeoffs between opposing values. This is the aim of the current research.

\section{The Structure of Basic Values}

The value theory posits that basic values form a circular continuum that reflects the motivational conflict or compatibility among them (Schwartz, 1992). Values are compatible if decisions and behaviors that express or promote the goals of one also express or promote the goals of the other. Values conflict if decisions or behaviors that express or promote the goals of one do so at the expense of the other. The more compatible any two values, the closer they are on the circle, the more in conflict, the more distant. Figure 1 displays the theorized value circle, modified to present the order that Schwartz et al. (2012) found with an earlier instrument. We expect to replicate this order in our four new samples.

Figure 1 identifies three bases for the order of values around the motivational circle. These bases help to explain why specific values promote or oppose particular behaviors. The outermost circle distinguishes values that concern ways of coping with anxiety and protecting the self (bottom) from values that concern relatively anxiety-free ways in which people grow and expand the self (top). The second circle distinguishes values concerned with personal outcomes (right) from values concerned with outcomes for others or for established institutions (left). The inner circle combines the values into four higher-order values that form two bipolar dimensions of motivationally incompatible values, self-transcendence versus self-enhancement and conservation versus openness to change. As Figure 1 shows, it is possible to measure 19 values, to combine adjacent values to measure the original 10 values (plus humility and face, added in the refined theory), or to measure the four higher-order values, as appropriate to one's research purposes.

The current study goes beyond previous research in several important ways. First, previous research on

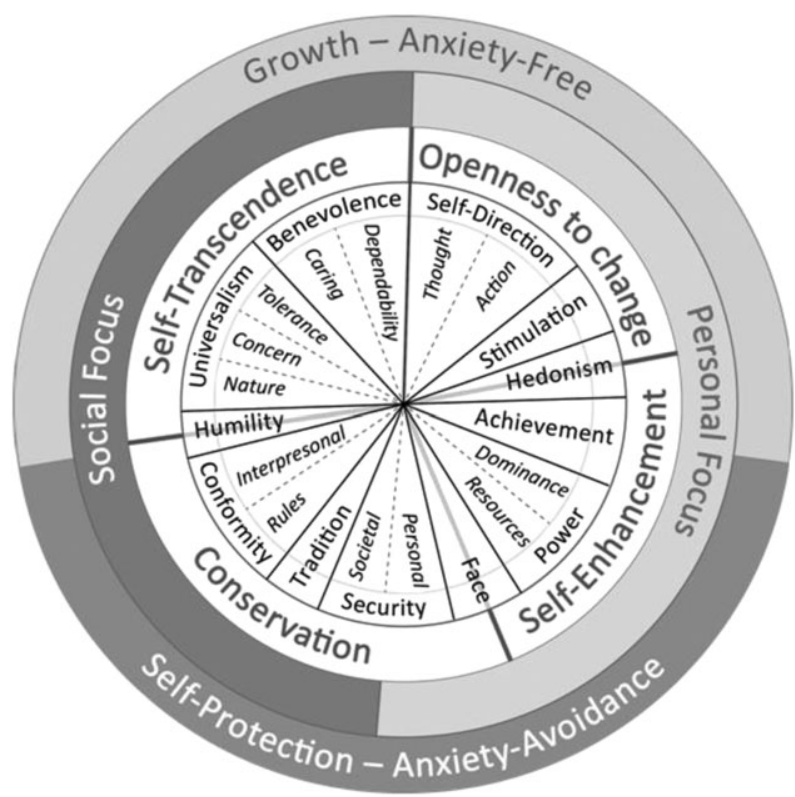

Fig. 1: Circular motivational continuum of 19 values with sources that underlie their order 
value-behavior relations examined relations of each value only to behaviors it was expected to promote (Bardi \& Schwartz, 2003) and/or to inhibit (Schwartz $\delta$ Butenko, 2014). In doing so, it ignored a key postulate of the value theory inherent in the notion of the motivational circle: Any behavior is a product of tradeoffs between the values that promote and oppose it. If a value on one side of the circle promotes a particular behavior, values on the opposing side of the circle should simultaneously inhibit that behavior. We examine the tradeoff between opposing values in predicting each behavior.

Second, as noted, Schwartz and Butenko (2014) used self-reports of values and behavior. This may increase value-behavior consistency because people may base self-reported values on their recall of past behaviors. Moreover, self-reports suffer from several response biases (e.g., Mõttus et al., 2012; Paulhus \& Vazire, 2009). We reduced such biases by using both self-reports and other-reports of behavior. The advantages of each type of report may compensate, at least partly, for the disadvantages of the other. The main advantage of self-reports is that individuals base them on the full range of their experiences; raters can base otherreports only on experiences they share with the target or on hearsay. The main disadvantage of self-reports is that such motivated biases as social desirability and consistency seeking distort them; other-reports are less vulnerable to these biases (John \& Robins, 1993; Paulhus \& Vazire, 2009). Other-reports of values, like other-reports of personality, may explain unique variance in behavior (Vazire \& Mehl, 2008; Wagerman \& Funder, 2007). We sought to utilize the unique information available in both self-reports and otherreports of behavior by combining them into composite scores rather than treating them as indicators of a latent variable, which would capture only the variance they share.

Third, we examine characteristics of values and of behavior that may moderate the strength of valuebehavior relations. In a study in Israel, Bardi and Schwartz (2003) found that highly endorsed values and frequently performed behaviors exhibited weaker value-behavior relations. They suggested that such values and behaviors are normative and proposed that normative pressure induced individuals to comply with group expectations rather than to base their behavior on their own values. This yielded weaker value-behavior correlations for normative than for non-normative values and behavior. Tens of subsequent publications have cited this finding (e.g., Fischer et al., 2009; Hitlin \& Piliavin, 2004; Lipponen, Bardi, \& Haapamäki, 2008; Lönnqvist, Leikas, Paunonen, Nissinen, \& Verkasalo, 2006) without testing it. However, it did not replicate in the one Russian study that did test it with selfreported behavior (Schwartz \& Butenko, 2014). We test this widely cited normative explanation of variation in the strength of value-behavior relations for the first time across multiple countries using self-reported and other-reported behavior.
Gender is another potential moderator of the strength of value-behavior relations. The normative pressure explanation implies that relations should be stronger when gender role expectations are weak, say for power for women and benevolence for men (e.g., Best $\delta$ Thomas, 2004; Eagley, Wood, \& Johannesen-Schmidt, 2004; Schwartz \& Rubel, 2005). Another mechanism yields the same prediction. It combines gender roles with the idea that value-behavior relations are stronger when the decision to behave is more deliberate and consciously controlled (Roccas, Sagiv, Schwartz, \& Knafo, 2002). Self-conscious deliberation is more likely before engaging in role-inappropriate behavior. Hence, value-behavior relations should be stronger for power for women and benevolence for men. Schwartz and Butenko (2014) found that relations of power values to behavior were indeed stronger for women than for men. However, they found no gender differences for benevolence values and several unexpected gender differences. We evaluate the validity and generalizability of the normative and gender role explanations by examining gender differences in the strength of value-behavior relations in four countries.

\section{Hypotheses}

To validate the refined theory, it is necessary to determine whether each of the 19 values in the theory relates as expected to behavior. Although multiple values may motivate a single behavior, some types of behavior are likely to be motivated primarily by one value and inhibited by its conceptually opposed values. For example, behaviors that manipulate or control others for one's own benefit are likely to be motivated by power values and inhibited by self-transcendence values. In order to assess value-behavior relations, we specified in advance a set of behaviors we expected to be linked most strongly with each of the 19 values.

We assess the predictive, convergent, and discriminant validity of the 19 values by investigating how each one relates to its corresponding behavior as well as to the behaviors corresponding with the other 18 values. We also examine the tradeoffs between each value and its opposing values in predicting the corresponding behavior. Each of the following hypotheses actually specifies 19 testable hypotheses, one for each value.

la. Values correlate significantly positively with their a priori corresponding behavior.

1b. Values correlate more positively with their corresponding behavior than with any other behavior.

2a. Behaviors correlate significantly positively with their corresponding value.

2b. Behaviors correlate more positively with their corresponding value than with any other value.

These hypotheses express the theoretical view that each of the 19 values has unique positive associations with behavior, so it is desirable to distinguish among them. The following hypotheses address the claim that 
there is a tradeoff between values that propel and inhibit behaviors. The set of higher-order values from the opposing side of the value circle represents the inhibiting values. We use a set of potentially inhibiting values rather than any single value because more than one value is motivationally opposed to each value, at least in part.

3. The higher-order values correlate negatively with the behaviors motivated by a value to which they are motivationally opposed.

4. When jointly predicting a behavior by its corresponding value and opposing higher-order values, both contribute significantly, the former positively and the latter negatively.

Hypotheses 1, 2, 3, and 4 test the theoretical assumption that behavior entails a tradeoff between values that propel the behavior and those that oppose it (Schwartz, 2010). Hypothesis 5 tests the implications for valuebehavior relations of the theoretical assumption that values form a circular motivational continuum.

5. The relative magnitude of the correlations of each value with the 19 behaviors and of each behavior with the 19 values approximates a sinusoid curve reflecting the order of the circular motivational continuum.

We test these five hypotheses for all 19 values.

The five hypotheses address the primary objective of this study, to assess the validity of the 19 values as unique predictors of behavior. We also assessed the expectations that normative pressure and gender moderate the strength of value-behavior relations. Before addressing the hypotheses, however, we addressed two critical, preliminary questions: (i) Can we discriminate each of the 19 values in the data from each country?; (ii) Are the discriminated values ordered around the value circle as theorized (i.e., as in Figure 1)?

This study does not assess causality. Nonetheless, there is reason to believe that value-behavior associations attest to at least some causal influence of values on behavior. Studies that prime values support such causal influence by showing that value manipulations influence subsequent behavior in value-consistent directions (e.g., Maio, 2015; Maio, Pakizeh, Cheung, \& Rees, 2009; Sagiv, Sverdlik, \& Schwarz, 2011; Verplanken \& Holland, 2002). For example, priming achievement values improved task performance, priming benevolence values increased volunteering, and priming self-direction values promoted information seeking. Values may lead to behavior through both motivational and cognitive processes. As expressions of underlying motivations in the form of goals, values make behavior that promotes these goals more attractive and motivates such behavior (Feather, 1995; Roccas \& Sagiv, 2010; Rokeach, 1973). As mental representations of desirable goals, values promote behaviors that are cognitively associated with and instantiate these goals (Maio, 2015). In both instances, activation of a value may give rise to an affective response that energizes action (Schwartz, 2015).

\section{Method}

\section{Participants}

The samples included 1857 respondents from Italy, Poland, Russia, ${ }^{1}$ and the USA. Table 2 presents basic demographic information. These were convenience samples that varied in size and in composition by population, age, gender, and education. Because of these and other differences among the samples, it is appropriate to view this research as four separate replications. In all countries, participation was voluntary, participants received no compensation and were free to withdraw, and researchers guaranteed anonymity in reporting the data. Ethical clearance was obtained from Institutional Review Boards in Italy and the USA. Institutional Review Boards were not consulted in Poland or Russia, where questionnaire research like this, that poses no risks to the adult, volunteer participants, requires no clearance.

\section{Procedure}

Pairs of respondents completed a questionnaire that measured basic values and everyday behaviors. They first reported their own values, then rated the everyday behaviors of the person they chose as a partner, completed a distraction task, and then rated their own everyday behaviors. ${ }^{2}$ The two persons responded at the same time, without consultation. Sessions took approximately 40 minutes. We presented the research as a study of interpersonal familiarity.

In both Italy (Rome) and Poland (Warsaw area), trained university students recruited pairs of adult members of the general population among their acquaintances and friends of acquaintances. Data were gathered in respondents' homes. The Polish students received course credit for their work. In Russia (Moscow), trained graduate students recruited and administered the questionnaire to more than half the student respondent pairs in classrooms, one-third in dormitories, and the remainder online. In the USA (Florida), the researcher recruited pairs of students in classrooms where they completed the questionnaires or took them home

\footnotetext{
${ }^{1}$ The self-report data of the Russian sample were used by Schwartz and Butenko (2014). We include both self-reports and other-reports of behavior, test additional hypotheses, and analyze the data differently. ${ }^{2}$ We chose this set order of tasks rather than counterbalancing based on findings in an earlier study. Fifteen respondents reported their values prior to their behaviors, separated by a distraction task, and 15 answered in the reverse order. Value-behavior correlations were substantially stronger when behaviors were measured first. This suggests that people are more likely to infer their values-largely unarticulated latent constructs-from their concrete behaviors and seek consistency of self-reports than they are to infer their behaviors —which they have observed directly-from their values. Consequently, placing values first introduces less of this unwanted source of variance in value-behavior relations.
} 
Table 2. Description of the samples in the study

\begin{tabular}{llcclccc}
\hline Country & Sample type & $N$ & \% female & Language & Age M(SD) & Education years M(SD) \\
\hline Italy & Adults & 300 & 56 & Italian & $32.9(13.8)$ & Mode & 14.4 (3.1) \\
Poland & Adults & 1218 & 50 & Polish & $32.3(11.2)$ & $15.5(2.6)$ & written \\
Russia & Students & 266 & 68 & Russian & $20.7(4.0)$ & $13.7(2.0)$ & $95 \%$ written $5 \%$ online \\
United States & Students & 232 & 59 & English & $24.2(7.6)$ & $14.8(2.0)$ & $90 \%$ written 10\% email \\
\hline
\end{tabular}

and returned them to course instructors. Some $10 \%$ of respondents from an online course responded by email. Respondents were requested to choose as a partner someone they knew quite well.

\section{Measures $^{3}$}

Values. Respondents completed a revised version of the Portrait Values Questionnaire (PVQ) used by Schwartz et al. (2012). This version, the PVQ-Revised $(\mathrm{R})$, replaced the nine items identified as problematic in that study and reordered the wording of some other items. Like the previous version, the PVQ-R includes 57 short, gender-matched, verbal portraits of different people, each describing a goal that is important to the person. For each portrait, respondents indicate how similar the person is to themselves on a 6-point scale: 1-not like me at all, 2-not like me, 3-a little like me, 4-moderately like me, 5-like me, and 6-very much like me.

Respondents' own values are inferred from the values of the people they describe as similar to themselves. For example, a respondent who indicates that a person described by "Enjoying life's pleasures is important to him" is similar to himself presumably attributes importance to hedonism values. Additional examples are, "Obeying all the laws is important to her" (conformity-rules) and "It is important to him never to be humiliated" (face). Online Supplement A presents the full list of PVQ-R items. For each language, iterations of translation and back-translation to the English original were carried out until agreement was reached that the translation optimally captured the nuances of each item.

Behaviors. We adopted the 85-item Everyday Behavior Questionnaire (EBQ) developed by Schwartz and Butenko (2014). It consists of sets of three to six behaviors that are expected to be motivated most strongly by one of the 19 values in the theory. The items were originally chosen based on a pretest of 116 items with a sample of 25 Russian students who were familiar with the 19 values. We asked these students to assign each behavior to the value to which it was most relevant. We included in the EBQ only those behaviors that at least two-thirds of the pretest respondents assigned to the expected value and at least $80 \%$ assigned to that value or a value adjacent to it in the circle. The number

\footnotetext{
${ }^{3}$ The anonymized raw data, related coding information, and all materials used to collect data in their original wording are available through the Social Science Data Center of the Hebrew University of Jerusalem.
}

of items for each value reflects the diversity of contexts and of actions in which people commonly express that value. More than 95\% of Schwartz and Butenko's (2014) Russian respondents had reported opportunities to perform each behavior during the past year. The researchers in the countries of this study judged that opportunities to perform these behaviors were also common in their countries, a judgment subsequently confirmed by participants' responses.

The EBQ uses the act-frequency approach of Buss and Craik (1983), who argued that the best way to assess a tendency to behave in particular ways is to measure the frequency of actions across time and situations. The EBQ asks respondents how frequently they or their target person have performed each behavior in the past year. Gosling, John, Craik, and Robins (1998) have shown that reports of the frequency of highly specific behaviors are often quite accurate. Respondents estimated how often they [the other] have engaged in each behavior during the past year relative to the number of times they [he or she] had an opportunity (emphasis in the original) to do so. The response scale was numbered and labeled as follows: 0-never, 1-rarely (about a quarter of the times), 2-sometimes (about half of the times), 3-usually (more than half the times), and 4-always. An additional response alternative, "never had even one opportunity to do something like this," allowed respondents to distinguish never performing a behavior from never having an opportunity to do so. We treated this response alternative, which constituted less than $1 \%$ of responses, as missing data.

Online Supplement B lists all of the behavior items in the EBQ. Examples, with their corresponding value, are "learned something simply for the joy of learning" (selfdirection- thought), "kept promises I made to friends or family" (benevolence-dependability), and "celebrated national or ethnic group holidays" (tradition). We employed the same translation-back-translation procedure used for values to translate the EBQ.

Distractions. After reporting their own values and rating the 85 behaviors of their target person, respondents completed two distraction tasks before rating their own behaviors. This was intended to reduce recall of self-reported values that might bias self-ratings of behavior toward consistency. Respondents first completed a colorful, relatively easy maze. They were then asked to compose as many words as possible from the letters in the word for 'medicine' in their native language (e.g., медицина in Russian). The distraction tasks took 5-10 minutes. 
Familiarity. Lastly, respondents reported how well they knew their target person on a 5-point scale (1-not so well to 5-very well) and for how long. Mean familiarity ratings were $3.6(S D=1.03)$ in Russia, $4.4(S D=.97)$ in Italy, $4.4(S D=.72)$ in Poland, and 4.4 $(S D=.75)$ in the USA. These ratings indicate that respondents knew one another quite well. Mean length of acquaintance ranged from 2.45 years $(S D=2.81)$ in Russia to $14.63(S D=12.03)$ in Italy, and $82 \%$ of respondents had known their partners for over 3 years.

\section{Statistical Analyses}

Multi-group confirmatory factor analysis. We used multi-group confirmatory factor analysis (MGCFA) to assess distinctiveness and establish indices for the 19 values and the 19 sets of behavior. We followed the recommendation in Cieciuch and Schwartz (2012) and ran separate models for each higher-order value for the following reasons: (i) the number of respondents in all but the Polish sample was too small to assess models with 19 latent variables and 57 value items or 85 behavior items reliably (Harrington, 2008); (ii) the circularity of the values model means that value items might have negative cross-loadings on values on the opposite side of the motivational circle. CFA models of the whole circle might therefore introduce negative cross-loadings that would contribute to misspecification and reduce model fit (Davidov, Schmidt, \& Schwartz, 2008). Negative crossloadings on values on the opposite side of the circle are relevant for assessing the shape of the circular structure. They are irrelevant, however, to our goal of obtaining cross-culturally comparable indicators for specific values and sets of behavior; (iii) this strategy is therefore the common practice for assessing the distinctiveness of single constructs in complex values models (e.g., Cieciuch \& Schwartz, 2012; Saris, Knoppen, \& Schwartz, 2013; Schwartz \& Butenko, 2014).

We assessed metric measurement invariance (equal loadings across groups) because that level of invariance is needed to compare correlations across countries, (Davidov, Meuleman, Cieciuch, Schmidt, \& Billiet, 2014). If full metric measurement invariance was not established, we tested for the partial invariance by releasing the most non-invariant loadings. Partial invariance is sufficient for meaningful cross-country comparisons when the loadings of at least two indicators per construct are equal across groups (Byrne, Shavelson, \& Muthen, 1989).

To evaluate model fit at the configural level, we used the comparative fit index (CFI), the root mean square error of approximation (RMSEA), and the standardized root mean square residual (SRMR). We treated CFI values $=>.90$, RMSEA values $<=.08$, and SRMR values $<=.08$ as indicating a reasonable model fit (Hu \& Bentler, 1999; Marsh, Hau, \& Wen, 2004). To evaluate metric measurement invariance, we applied criteria proposed by Chen (2007): Lack of invariance is indicated by a change larger than .01 in CFI, supplemented by a change larger than .015 in RMSEA or a change larger than .03 in SRMR, compared with the configural invariance model. We performed all analyses with Mplus 7.1 (Muthén \& Muthén, 1998-2012), using the maximum likelihood estimator.

We view basic values as latent variables that influence responses to specific value items. We therefore derived factor scores for each value from MGCFA at the metric measurement invariance level. Factor scores provide more reliable indices of values than the sum scores used in previous value-behavior research. We also derived factor scores for each of the 19 behaviors from the MGCFA at the metric level of measurement invariance of the set of a priori behavior items intended to tap it. We did this separately for self-reported and otherreported behavior and combined the two factor scores into a composite behavior score.

Multidimensional scaling. In order to assess the structure of relations among the 19 values and their order around the circular motivational continuum, we used confirmatory, non-metric, multidimensional scaling (MDS; Borg, Groenen, \& Mair, 2013). MDS provided graphic representations of the spatial relations among the 19 values, allowing us to assess whether they were ordered as theorized around the circle. The MDS spatial projections of the 19 values also disclosed whether it was possible to combine adjacent values to recapture the original 10 values and the four higherorder values. We ran separate MDS analyses in each country, using as input the factor scores for each value. For these analyses, we used the sPss 20 MDS Proxscal program (Chicago, IL, USA), with ordinal proximity transformations, Euclidian distance measures, and $Z$-score transformations of values. We also generated an overall structure by running an MDS analysis on the pooled within-sample covariance matrices of the four samples, equally weighting each sample. All analyses used a custom initial configuration (Bilsky, Janik, \& Schwartz, 2011) derived from the theorized circle in Figure $1 .{ }^{4}$ We compared the structure in each of the four samples to the overall structure by rotating it to the overall structure with orthogonal Generalized Procrustes Analysis (Commandeur, 1991).

Regression. In order to formally test the hypothesis that the value expected to propel a behavior and the higher-order value opposed to it jointly predict the behavior, the former positively and the latter negatively, we ran separate regressions for each behavior in each sample. Specifically, we regressed the centered factor score of each behavior on the corresponding centered value and the centered opposing higher-order value. We used the opposing higher-order value rather than a specific value from the opposite side of the

\footnotetext{
${ }^{4}$ The design matrix for the initial configuration assigned starting coordinates for each of the 19 values at increasing angles of $19^{\circ}$ (i.e., $19 \times 19 \sim 360$ ). Results were very similar using Torgerson initial configurations.
} 
motivational circle for two reasons. Values are not necessarily evenly spaced around the circle, so it is not certain, which is the most opposed value. Also, multiple values oppose each value in the circle motivationally and are likely to inhibit the behaviors it propels. To estimate the overall effects on each behavior across the four diverse samples, we used hierarchical linear modeling (HLM 6; Raudenbush \& Bryk, 2002). This method takes the hierarchical structure of the data into account. We centered the predictors on the group means in order to examine effects within each sample and to avoid confounding by mean differences between samples on the predictors. The HLM analyses also revealed whether the effects of values on behaviors varied significantly across samples.

\section{Results}

\section{Preliminary Issues: Discrimination of the Values and Behaviors}

Before testing the hypotheses, it was necessary to assess whether the 19 values and behaviors could be discriminated and to develop indices for them. The three panels of Table 3 report goodness-of-fit statistics for the MGCFA models of values, self-rated behaviors, and

Table 3. Global fit measures for the continuous multi-group confirmatory factor analyses across four countries: tests of invariance

\begin{tabular}{|c|c|c|c|c|c|c|c|}
\hline Values & \# factors (items) & $\chi^{2}$ & $d f$ & RMSEA & SRMR & $\mathrm{CFI}$ & Items dropped \\
\hline Self-transcendence & $5(15)$ & & & & & & \\
\hline Configural & & 719.4 & 268 & $.058[.053-.063]$ & .042 & .953 & \\
\hline Metric & & 809.0 & 295 & $.059[.054-.064]$ & .057 & .947 & bed2 \\
\hline Self-enhancement & $4(12)$ & & & & & & \\
\hline Configural & & 897.01 & 188 & $.086[.081-.092]$ & .063 & .906 & \\
\hline Metric & & 1008.7 & 212 & $.086[.081-.092]$ & .078 & .895 & \\
\hline Partial metric ${ }^{a}$ & & 982.8 & 209 & $.086[.080-.091]$ & .073 & .900 & \\
\hline Conservation & $6(18)$ & & & & & & \\
\hline Configural & & 1460.0 & 480 & $.064[.060-.067]$ & .053 & .915 & \\
\hline Metric & & 1545.6 & 516 & $.063[.059-.067]$ & .059 & .911 & \\
\hline Openness & $4(12)$ & & & & & & \\
\hline Configural & & 445.0 & 116 & .075 [.068-.082] & .048 & .927 & \\
\hline Metric & & 540.3 & 134 & $.078[.071-.084]$ & .069 & .910 & he3 sda3 \\
\hline Partial metric ${ }^{b}$ & & 508.9 & 128 & $.077[.070-084]$ & .065 & .916 & \\
\hline \multicolumn{8}{|l|}{ Behavior self-rated } \\
\hline Self-transcendence & $5(21)$ & & & & & & \\
\hline Configural & & 1403.5 & 500 & $.060[.056-.064]$ & .054 & .915 & unt3 bec 2 bed3 \\
\hline Metric & & 1535.7 & 539 & $.061[.057-.064]$ & .062 & .906 & \\
\hline Self-enhancement & $4(16)$ & & & & & & \\
\hline Configural & & 566.0 & 192 & $.062[.056-.068]$ & .050 & .917 & ac1 ac2 fac2 \\
\hline Metric & & 632.4 & 216 & $.062[.056-.067]$ & .058 & .907 & fac3 \\
\hline Conservation & $6(28)$ & & & & & & sep3 sep4 sep6 \\
\hline Configural & & 1273.9 & 612 & $.046[.043-.050]$ & .048 & .900 & ses 1 cor 1 coi 1 co14 hum 2 hum 3 \\
\hline Metric $^{c}$ & & 1373.5 & 654 & $.047[.043-.050]$ & .053 & .892 & \\
\hline Openness & $4(19)$ & & & & & & \\
\hline Configural & & 710.7 & 284 & $.055[.050-.060]$ & .053 & .904 & sda1 sda3 st4 he4 he5 \\
\hline Metric & & 814.3 & 314 & $.056[.051-.061]$ & .059 & .887 & \\
\hline Partial metric ${ }^{d}$ & & 766.4 & 305 & $.055[.050-060]$ & .056 & .896 & \\
\hline \multicolumn{8}{|l|}{ Behavior other-rated } \\
\hline Self-transcendence & $5(21)$ & & & & & & \\
\hline Configural & & 1568.4 & 568 & $.059[.056-.063]$ & .058 & .906 & bec1 bed3 \\
\hline Metric & & 1667.5 & 610 & $.059[.055-.062]$ & .063 & .900 & \\
\hline Self-enhancement & $4(16)$ & & & & & & \\
\hline Configural & & 543.4 & 192 & $.060[.054-.066]$ & .052 & .904 & ac1 ac2 fac2 \\
\hline Metric & & 592.1 & 216 & $.059[.053-.064]$ & .058 & .898 & fac4 \\
\hline Conservation & $6(28)$ & & & & & & sep 3 ses 3 cor3 \\
\hline Configural & & 1529.9 & 860 & $.039[.036-.042]$ & .045 & .907 & cor3 coi1 hum2 \\
\hline Metric & & 1637.3 & 911 & $.040[.037-.043]$ & .049 & .900 & \\
\hline Openness & $4(19)$ & & & & & & \\
\hline Configural & & 494.4 & 228 & $.048[.042-.054]$ & .045 & .922 & sdt1 sda2 sda3 st3 he4 he5 \\
\hline Metric & & 593.5 & 255 & $.051[.046-.057]$ & .053 & .900 & \\
\hline Partial metric ${ }^{\mathrm{e}}$ & & 520.7 & 240 & $.048[.043-.054]$ & .047 & .917 & \\
\hline
\end{tabular}

${ }^{a}$ Added correlated error between por1-por2 and released loading of ac1; ${ }^{b}$ Released loadings of sd1 and st1.

${ }^{c}$ Added correlated errors between tr2-tr3 and between coi1-coi3.

dReleased loadings of st1, he1, sda4.

${ }^{\text {e}}$ Added correlated errors between st1-st2 and between he2-he3 and released loadings of sdt2, sdt3, sda4, st4, he1.

Note: CFI, comparative fit index; RMSEA, root mean square error of approximation; SRMR, standardized root mean square residual. If loadings were released in a partial metric model, at least two loadings remained constrained for each factor. 
other-rated behaviors. The first column lists the number of domains (factors) in each higher-order domain and the number of items in the CFA model tested. The table provides the statistics for the configural and metric invariance models, and, if the fit of the metric model was only borderline, the partial metric model. The table also notes any items that were dropped, loadings that were released, and correlated errors within domain, based on the modification indices. We constrained all models to have no cross-loadings across factors. All models met at least two of the three criteria for an adequate fit.

The top panel of Table 3 presents findings for the basic values CFA models. At least two of the three a priori items served as indicators of each latent value factor. The middle panel presents findings for the self-rated behaviors models. The factor scores for the latent self-rated behaviors were all based on at least three a priori items. The bottom panel presents findings for the CFA models of other-rated behaviors. The factor scores for the latent other-rated behaviors were also all based on at least three a priori items.

Correlations between self-rated and other-rated behaviors averaged $.43(S D=.076)$ across behaviors and countries, ranging from .29 for self-direction-action behaviors to .61 for tradition behaviors. These correlations are somewhat higher than those reported in studies of personality traits (e.g., McCrae $\&$ Costa, 1987; Mount, Barrick, \& Strauss, 1994) and of act frequencies (summarized in Gosling et al., 1998). Supplement C reports the correlations for the 19 behavior indices in each country. Based on these results, we averaged the self-rated and other-rated behavior factor scores to form the behavior indices. We also ran separate analyses of value-behavior relations for the self-ratings and other-ratings to insure that the pattern of findings was the same. This was indeed the case. ${ }^{5}$

Discrimination of both values and behaviors in 19 domains enabled us to test the hypotheses regarding unique value-behavior associations for each of the 19 values. We used the factor scores from the MGCFAs to operationalize the values and behaviors in subsequent analyses.

\section{Preliminary Issues: The Circular Structure of Relations Among the Values}

Hypotheses 3, 4, and 5 assume that the values are ordered around the circle as the theory specifies. Figure 2 presents the MDS plot of the 19 values based on the pooled correlation matrix for the four countries. It corresponds exactly to the theorized order in Figure 1. The separate MDS plots for each sample revealed structures quite similar to the combined sample (Supplement G). Column 1 of Table 4 presents the Spearman correlation between the order of the values around the circle and the theorized order in each sample. Reflecting only one or two reversals of adjacent values, all correlations were .99 . This made it feasible to

\footnotetext{
${ }^{5}$ Not unexpectedly, correlations were stronger in most instances for self-rated behavior
}

\section{Openness to Change}

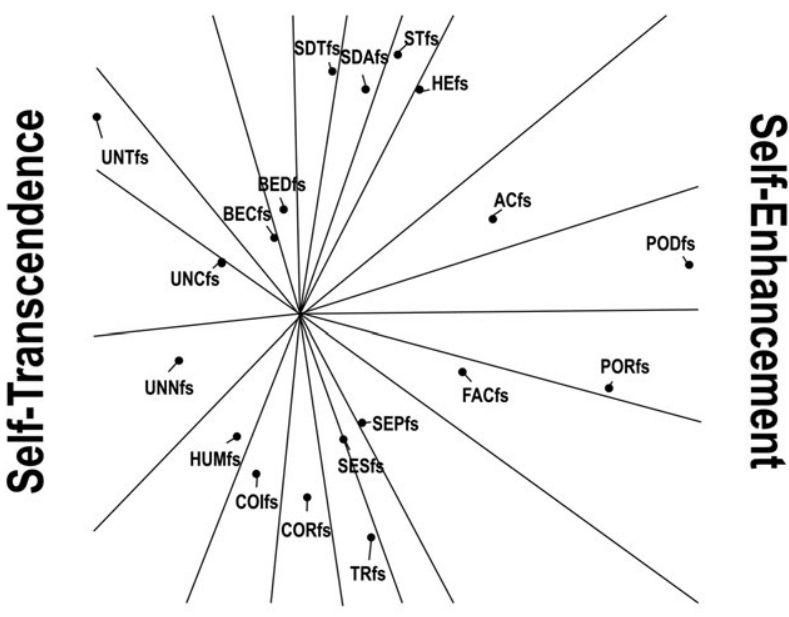

\section{Conservation}

Fig. 2: Multidimensional scaling analysis of 19 values based on the pooled within-sample covariance matrix for four countries. $N=1857$, Stress $1=.1$, dispersion accounted for $=.99$, Tucker's coefficient of congruence $=.99$.

Note: $\mathrm{fs}=$ factor score, $\mathrm{SDT}=$ self-direction-thought, the remaining value labels going counterclockwise around the circle from SDT follow the order in Table 1 and Figure 1.

Table 4. Comparisons of value structures in samples from four countries with the overall value structure

\begin{tabular}{lcc}
\hline & $\begin{array}{c}\text { Spearman correlation between } \\
\text { country order and overall order of } \\
19 \text { values }\end{array}$ & $\begin{array}{c}\text { Tucker's phi dimension } \\
\text { 1/dimension } 2\end{array}$ \\
\hline Poland & .99 & $.92 / .97$ \\
Italy & .99 & $.95 / .90$ \\
USA & .99 & $.98 / .94$ \\
Russia & .99 & $.92 / .96$ \\
\hline
\end{tabular}

test the hypotheses regarding sinusoidal associations between values and behaviors. In every country, the six conservation values opposed the four openness-tochange values and the four self-enhancement values opposed the five self-transcendence values. This made it feasible to use the higher-order values to test the hypothesized tradeoffs between opposing values in predicting behavior.

Column 2 of Table 4 presents the Tucker's phi coefficients obtained when applying Generalized Procrustes Analysis (Commandeur, 1991) to compare the structure in each sample to the overall structure. The coordinates of the items in each sample correlated at least .90 with those in the overall structure on both the first and second dimensions. This suggests a high level of similarity in the value structures (Van de Vijver \& Poortinga, 1994).

\section{Predictive and Discriminant Validity of the 19 Values}

Table 5 presents the pooled correlation matrix of each value with each behavior, equally weighting each sample, using centered factor scores. The correlations 


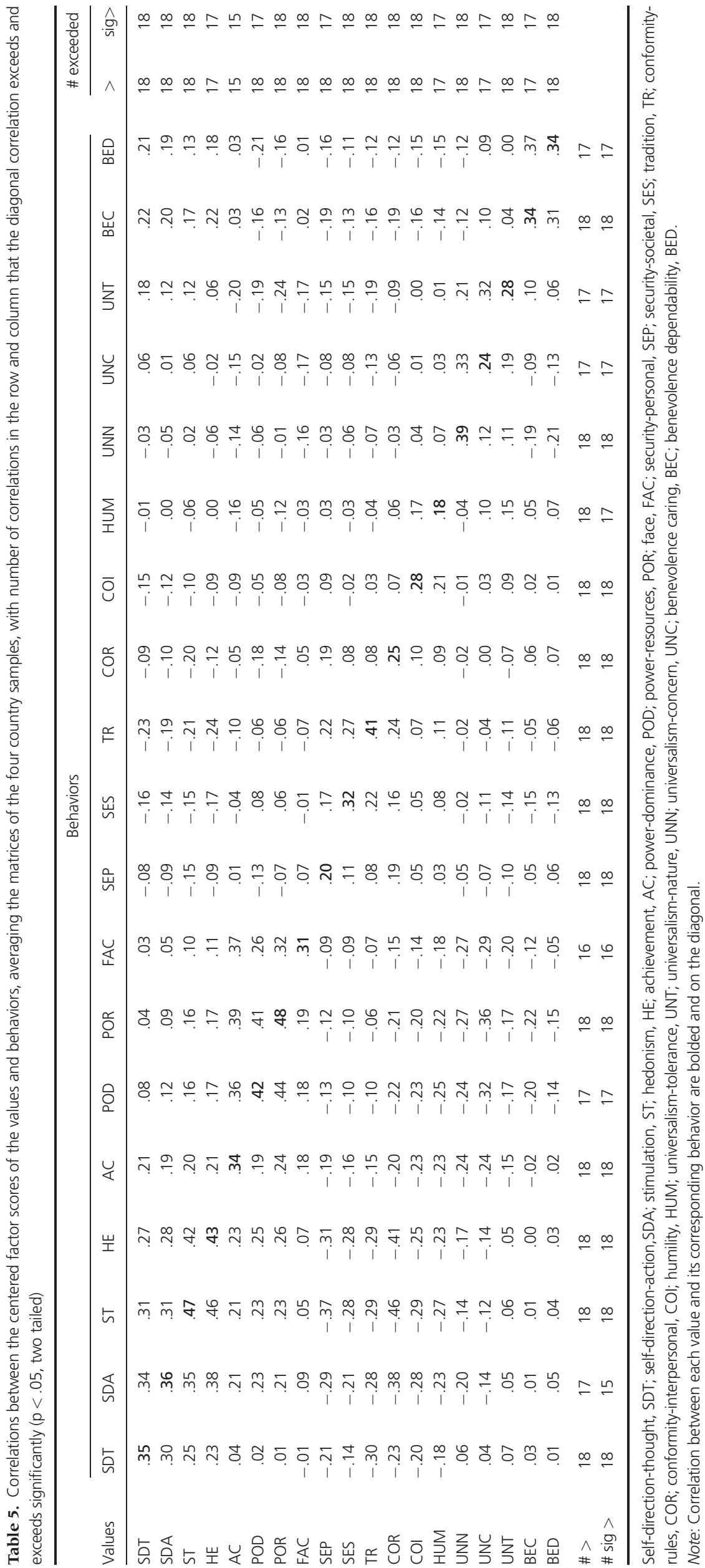


between each value and its corresponding behavior are on the diagonal. All these correlations were significant $(p<.001$, two tailed). According to hypothesis la (Hla), each value should correlate most positively with its corresponding behavior. This was the case for 13 of the 19 correlations: they exceeded the 18 correlations with other behaviors (see next to last column). The correlations for five other values exceeded 17 of the 18 other correlations, correlating more positively only with a behavior that corresponded with an adjacent value in the motivational circle. Given that we expect adjacent values to correlate similarly with other variables, this was a minor deviation. Thus, Hla received substantial support for $18 / 19$ values. The correlation of the achievement value with its corresponding behavior exceeded its correlations with only 15 other behaviors. Supplement D provides the correlation matrix for each country. These matrices also supported Hla. Seventy-four of the $76 \quad(4 \times 19)$ correlations, fully 97\%, were significant.

According to hypothesis $1 \mathrm{~b}$, each value should correlate significantly and more positively with its corresponding behavior than with any other behavior. The last column of Table 5 lists the number of correlations that the corresponding value-behavior correlation exceeded significantly ( $p<.05$, two tailed), comparing dependent correlations with Steiger's Z (1980). This correlation exceeded all other correlations for 12 values and all but one other correlation for six values. The one correlation not exceeded was with a behavior that corresponded with an adjacent value in the motivational circle. Overall, the correlations of values with their corresponding behavior exceeded their correlations with other behaviors in $98 \%$ of comparisons. Thus, hypothesis $1 \mathrm{~b}$ received substantial support for 18 of the 19 values. The correlation of the achievement value with its corresponding behavior significantly exceeded only 15 of the correlations with other behaviors. In the matrices for the four countries, correlations with the corresponding behavior exceeded those with all other behaviors in $95 \%$ of $1368(4 \times 18 \times 19)$ comparisons.

According to hypotheses $2 \mathrm{a}$ and $2 \mathrm{~b}$, each behavior should correlate most positively with its corresponding value, and this correlation should exceed its correlation with any other value. Comparing each of the correlations on the diagonal of Table 5 with the correlations in its column addresses these hypotheses. As indicated in the next to last row of the Table, 13 of these 19 correlations were the highest in their column. The correlations for five other behaviors exceeded 17 of the 18 other correlations, excepting only a correlation with a value adjacent in the circle to their corresponding value in four cases and two steps away in one case. The last row in the Table indicates that, for 12 behaviors, the correlation with its corresponding value exceeded all other correlations significantly ( $p<.01$, two tailed) and, for five behaviors, it exceeded all but one correlation with an adjacent value. Thus, both hypotheses $2 \mathrm{a}$ and $2 \mathrm{~b}$ received substantial support for 18 of the 19 behaviors, all but face behaviors. In the matrices for the four countries, correlations of behaviors with their corresponding value exceeded those with all other values in $96 \%$ of 1368 comparisons, 94\% significantly.

According to hypothesis 3, each of the 19 values should correlate negatively with the behaviors motivated by values to which that value is motivationally opposed-values distant in the motivational circle. To test this hypothesis, we examined the correlation of each value with the behaviors presumably motivated by the opposing higher-order values (e.g., the correlations of the power-dominance value with the behaviors presumably motivated by the self-transcendence values). Table 5 reveals that $95 \%$ of the correlations of a value with the behaviors that an opposing higherorder value presumably motivates were negative. The exceptions were near-zero correlations, ranging from .01 to .03 , of benevolence-caring and benevolencedependability behaviors with achievement and face values. In the separate matrices of the four countries, $93 \%$ of the correlations with behaviors motivated by opposing values were negative. These findings support hypothesis 3.

\section{Value Tradeoffs}

As in previous research on value-behavior relations (Bardi \& Schwartz, 2003; Schwartz \& Butenko, 2014), however, the preceding analyses do not address the key idea inherent in the notion of the motivational circle of values: Behavior is a product of tradeoffs between values that propel it and values that oppose it. This implies that the corresponding value and the opposing higher-order value jointly predict behaviors, the former positively and the latter negatively (hypothesis 4). Regressions that included both the values expected to propel and to inhibit each behavior provided a direct test of value tradeoffs.

Columns 1 to 8 in Table 6 present the resultant beta coefficients and zero-order correlations for each country. All correlations of values with their corresponding behaviors were positive and significant $(p<.05$, one tailed). All opposing higher-order values correlated negatively with the behaviors, as expected, and 71/76 were significant. All but one of the standardized beta coefficients for the corresponding values were in the expected positive direction, and 69/76 were significant. ${ }^{6}$ For the opposing higher-order values, $72 / 76$ betas were negative, as expected, but only 41 were significant.

The right side of Table 6 presents results of the HLM analyses, summarizing findings across the four samples. All 19 values predicted their corresponding behavior significantly. Eighteen of the betas for the opposing higher-order values were negative, as expected, and nine were significant. For benevolence-caring behavior, the beta of the self-enhancement higher-order value

${ }^{6}$ In the USA, the standardized beta for the self-direction-action value was -.11 , although its correlation was positive and significant (.12). 
Table 6. Multiple regressions of behaviors on corresponding value and opposing higher-order value and zero-order value-behavior correlations in four countries, based on centered factor scores from the multigroup confirmatory factor analysis

\begin{tabular}{|c|c|c|c|c|c|c|c|c|c|c|c|}
\hline \multirow{3}{*}{$\begin{array}{l}\text { Value-behavior domain } \\
\text { opposing higher order }\end{array}$} & \multicolumn{8}{|c|}{ Country } & \multirow{2}{*}{\multicolumn{2}{|c|}{$\begin{array}{c}\text { Combined } \\
\text { Samples } \\
N=2016\end{array}$}} & \multirow{3}{*}{$\begin{array}{c}\text { Do } \\
\text { slopes } \\
\text { vary? }\end{array}$} \\
\hline & \multicolumn{2}{|c|}{ Poland $N=1218$} & \multicolumn{2}{|c|}{ Italy $N=300$} & \multicolumn{2}{|c|}{ USA $N=232$} & \multicolumn{2}{|c|}{ Russia $N=266$} & & & \\
\hline & $\beta$ & $r$ & $\beta$ & $r$ & $\beta$ & $r$ & $\beta$ & $r$ & $\beta$ & $R^{2}$ & \\
\hline Self-direction thought (SDT) & $.35 * *$ & $.42 * *$ & $.24^{\star}$ & $.31 * *$ & $.21 * *$ & $.36 * *$ & $.20 * *$ & $.33 * *$ & $.25^{\star *}$ & .152 & no \\
\hline Conservation & $-.10 * \star$ & $-.34 * \star$ & $-.15^{\star}$ & $-.26^{\star}$ & $-.26 * *$ & $-.38 * *$ & $-.20 * *$ & $-.33 * *$ & $-.18 * *$ & & no \\
\hline Self-direction action (SDA) & $.19 * *$ & $.44^{\star *}$ & $.38 * *$ & $.52^{* *}$ & -.11 & $.12^{*}$ & $.22 * *$ & $.36 * *$ & $.16^{\star}$ & .195 & yes \\
\hline Conservation & $-.39 * *$ & $-.51 * *$ & $-.30 * *$ & $-.47 * *$ & $-.41 * *$ & $-.35^{\star *}$ & $-.21 *$ & $-.36 * *$ & $-.29 * *$ & & yes \\
\hline Stimulation (ST) & $.26^{\star \star}$ & $.49 * *$ & $.39 * *$ & $.60 * *$ & .06 & $.30 * *$ & $.36 * *$ & $.50 * *$ & $.29 * \star$ & .288 & yes \\
\hline Conservation & $-.37^{* *}$ & $-.53 * *$ & $-.37 * *$ & $-.60 * *$ & $-.40 * *$ & $-.44^{* *}$ & $-.14^{\star}$ & $-.38 * *$ & $-.34^{\star *}$ & & yes \\
\hline Hedonism (HE) & $.21 * *$ & $.45 * *$ & $.34 * *$ & $.53 * *$ & .08 & $.26 * *$ & $.38 * *$ & $.49 * *$ & $.28 * *$ & .294 & yes \\
\hline Conservation & $-.34^{* *}$ & $-.49 * *$ & $-.30 * *$ & $-.52 * *$ & $-.32 * *$ & $-.36 * *$ & $-.22 * *$ & $-.42 * *$ & $-.37 * *$ & & yes \\
\hline Achievement (AC) & $.40 * *$ & $.44^{\star *}$ & $.40 * \star$ & $.44^{* *}$ & $.23 * *$ & $.19 * *$ & $.23 * *$ & $.30 * *$ & $.17^{* *}$ & .130 & yes \\
\hline Self-transcendence & -.06 & $-.30 * \star$ & -.06 & $-.31 * *$ & .06 & -.05 & $-.13^{\star}$ & $-.25^{\star *}$ & -.04 & & yes \\
\hline Power-dominance (POD) & $.43^{* *}$ & $.54 * *$ & $.42 * *$ & $.50 * *$ & $.32 * *$ & $.37 * *$ & $.21 * *$ & $.27 * \star$ & $.23 * *$ & .213 & yes \\
\hline Self-transcendence & $-.24^{\star \star}$ & $-.44^{\star *}$ & $-.17^{\star *}$ & $-.36^{* *}$ & $-.21 * *$ & $-.28 * *$ & $-.18^{\star *}$ & $-.25^{\star *}$ & $-.34^{\star *}$ & & no \\
\hline Power-resources (POR) & $.52 * *$ & $.62^{q}$ & $.50 * *$ & $.55^{* *}$ & $.38 * *$ & $.46^{* *}$ & $.24 * *$ & $.30 * *$ & $.34 * *$ & .255 & yes \\
\hline Self-transcendence & $-.15^{* *}$ & $-.48^{* *}$ & -.08 & $-.38 * *$ & $-.15^{*}$ & $-.36 * *$ & $-.12^{*}$ & $-.25^{* *}$ & $-.16^{* *}$ & & no \\
\hline Face (FAC) & $.19 * *$ & $.29 * *$ & $.24^{* *}$ & $.33 * *$ & $.20 * *$ & $.26 * *$ & $.31 * *$ & $.35 * *$ & $.15^{* *}$ & .136 & no \\
\hline Self-transcendence & $-.30 * *$ & $-.36 * *$ & $-.34 * *$ & $-.41 * *$ & $-.13^{*}$ & $-.23 * *$ & -.10 & $-.24 * *$ & $-.22 * *$ & & yes \\
\hline Security-personal (SEP) & .04 & $.08^{* *}$ & $.37 * *$ & $.39 * *$ & $.20 * *$ & $.20 * *$ & .08 & $.14^{*}$ & $.15^{* *}$ & .043 & yes \\
\hline Openness to change & -.06 & $-.09 * *$ & -.04 & $-.20 * *$ & -.02 & $-.09 *$ & -.10 & $-.14^{*}$ & -.02 & & no \\
\hline Security-societal (SES) & $.32 * \star$ & $.35^{\star *}$ & $.38 * *$ & $.39 * *$ & $.19 * *$ & $.20 * *$ & $.32 * *$ & $.35 * *$ & $.28 * *$ & .098 & yes \\
\hline Openness to change & $-.07 *$ & $-.22 * *$ & -.03 & $-.21 * *$ & -.07 & $-.10 *$ & -.06 & $-.19 * *$ & $-.07^{\star}$ & & no \\
\hline Tradition (TR) & $.48 * *$ & $.52 * *$ & $.36 * *$ & $.45^{* *}$ & $.36 * *$ & $.41 * *$ & $.28 * *$ & $.28 * *$ & $.23 * *$ & .166 & yes \\
\hline Openness to change & $-.07^{\star}$ & $-.35^{\star *}$ & $-.20 * *$ & $-.35^{* *}$ & -.02 & $-.14^{\star}$ & -.01 & $-.17 * *$ & -.05 & & yes \\
\hline Conformity-rules (COR) & $.20 * *$ & $.20 * *$ & $.41 * *$ & $.42 * *$ & $.24 * *$ & $.26 * *$ & .11 & $.13^{*}$ & $.23 * *$ & .115 & yes \\
\hline Openness to change & -.00 & $-.13^{* *}$ & -.02 & $-.22^{* *}$ & -.06 & $-.16^{\star}$ & -.05 & $-.11 *$ & -.02 & & no \\
\hline Conformity-interpersonal (COI) & $.29 * \star$ & $.27 * *$ & $.27 * \star$ & $.32 * *$ & $.33 * *$ & $.32 * *$ & $.25 * *$ & $.22 * *$ & $.28 * *$ & .142 & no \\
\hline Openness to change & -.03 & $-.13^{* *}$ & $-.12^{*}$ & $-.22 * *$ & .02 & $-.14^{\star}$ & .06 & -.04 & -.02 & & yes \\
\hline Humility (HUM) & $.17 * *$ & $.13^{* *}$ & $.24 * *$ & $.29 * *$ & .08 & $.09 *$ & $.18^{* *}$ & $.19 *$ & $.18^{* *}$ & .112 & no \\
\hline ST, HE, AC, POD & $.06^{\star}$ & $-.03 * *$ & -.08 & $-.23 * *$ & -.03 & -.07 & -.01 & $-.09 *$ & -.01 & & no \\
\hline Universalism-nature (UNN) & $.22 * *$ & $.36 * *$ & $.51 * *$ & $.55^{* *}$ & $.36 * *$ & $.32 * *$ & $.29 * *$ & $.32 * *$ & $.23 * *$ & .147 & yes \\
\hline Self-enhancement & $-.09 * *$ & $-.15^{\star *}$ & $-.12^{*}$ & $-.13^{* *}$ & $.16^{\star}$ & -.06 & -.02 & $-.13^{* *}$ & -.02 & & yes \\
\hline Universalism-concern (UNC) & $.14^{\star *}$ & $.14^{\star *}$ & $.33 * *$ & $.41 * *$ & $.18 * *$ & $.25^{\star *}$ & $.15^{\star}$ & $.15^{\star}$ & $.19 * *$ & .113 & yes \\
\hline Self-enhancement & -.03 & $-.08 * *$ & $-.12^{\star}$ & $-.21 * *$ & -.06 & $-.10^{\star}$ & -.01 & $-.10^{\star}$ & -.03 & & no \\
\hline Universalism-tolerance (UNT) & $.20 * *$ & $.25^{* *}$ & $.26 * *$ & $.35^{* *}$ & $.20 * *$ & $.24 * *$ & $.25^{* *}$ & $.28 * *$ & $.23 * *$ & .155 & no \\
\hline Self-enhancement & $-.20 * *$ & $-.25^{* *}$ & $-.23 * *$ & $-.33 * *$ & $-.20 * *$ & $-.24 * *$ & $-.14^{*}$ & $-.21 * *$ & $-.13 * *$ & & no \\
\hline Benevolence caring (BEC) & $.37^{* *}$ & $.42 * *$ & $.37 * *$ & $.34 * *$ & $.37 * *$ & $.38 * *$ & $.23 * *$ & $.23 * *$ & $.29 * *$ & .144 & yes \\
\hline Self-enhancement & -.01 & $-.08 * *$ & .02 & -.07 & -.03 & $-.23 * *$ & .04 & -.01 & .03 & & no \\
\hline Benevolence dependability (BED) & $.45^{\star \star}$ & $.43^{\star *}$ & $.32 * *$ & $.32 * *$ & $.30 * *$ & $.32 * *$ & $.28 * *$ & $.28 * *$ & $.32 * *$ & .137 & yes \\
\hline Self-enhancement & -.02 & $-.10 * \star$ & -.01 & $-.12^{\star}$ & $-.18^{* *}$ & $-.24 * *$ & -.01 & $-.09 *$ & -.03 & & yes \\
\hline
\end{tabular}

${ }^{* *} p<.01$.

$* p<.05$, one tailed.

Combined samples analysis based on HLM.

Notes: Value and behavior scores are based on centered factor scores from confirmatory factor analyses. The higher-order value scores opposed to each value are based on the mean of the factor scores of the values opposed in the motivational value circle. The values that constitute each higher-order value are as follows: self-enhancement—AC, POR, POD, FAC; self-transcendence-UNN, UNC, UNT, BEC, BED; openness to change—SDT, SDA, ST, HE; and conservation-SES, SEP, TR, COR, COI. The set of values opposed to Humility is partly from the openness to change values and partly from the self-enhancement values.

Self-direction-thought, SDT; self-direction-action,SDA; stimulation, ST; hedonism, HE; achievement, AC; power-dominance, POD; power-resources, POR; face, FAC; security-personal, SEP; security-societal, SES; tradition, TR; conformity-rules, COR; conformity-interpersonal, COl; humility, HUM; universalism-tolerance, UNT; universalism-nature, UNN; universalism-concern, UNC; benevolence caring, BEC; benevolence dependability, BED

was .03. The variance in behavior accounted for by the tradeoff between values that propel and those that oppose the behavior averaged $16.0 \%$, ranging from $4.3 \%$ (personal security) to $29.4 \%$ (hedonism). The last column of Table 6 indicates that 14 of the 19 slopes of the propelling values and 9 of the 19 slopes of opposing higher-order values varied significantly across samples.

\section{Assessing the Sinusoid Curves}

Hypothesis 5 tests the implications for value-behavior relations of the claim that values form a circular motivational continuum. It posits that the correlations of each value with the 19 behaviors and of each behavior with the 19 values approximate a sinusoid curve that follows the order of the circular continuum. The strongest 
Table 7. Correlations approximating a sinusoid curve of the patterns of value-behavior correlations (based on the correlation matrix for the centered factor scores in Table 5)

\begin{tabular}{|c|c|c|}
\hline \multirow[b]{2}{*}{ Value-behavior domain } & \multicolumn{2}{|c|}{ Correlation approximating sinusoid curve for } \\
\hline & $\begin{array}{c}\text { Values with } 19 \\
\text { behavior sets }\end{array}$ & $\begin{array}{c}\text { Behavior sets with } \\
\qquad 19 \text { values }\end{array}$ \\
\hline Self-direction-thought & $.976 * * *$ & $.861 * * *$ \\
\hline Self-direction-action & $.921 * * *$ & $.895 * * *$ \\
\hline Stimulation & $.935 * * *$ & $.931 * * \star$ \\
\hline Hedonism & $.837 * * *$ & $.908 * * *$ \\
\hline Achievement & $.906 * * *$ & $.908 * * *$ \\
\hline Power-dominance & $.779 * * *$ & $.908 * * *$ \\
\hline Power-resources & $.802 * * *$ & $.821 * * *$ \\
\hline Face & $.715^{* * *}$ & $.632 * * *$ \\
\hline Security-personal & $.619 * * *$ & $.485^{\star}$ \\
\hline Security-societal & $.770 * * *$ & $.918 * * *$ \\
\hline Tradition & $.857 * * *$ & $.896 * * *$ \\
\hline Conformity-rules & $.928 * * *$ & $.769 * * \star$ \\
\hline Conformity-interpersonal & $.962 * * *$ & $.821 * * *$ \\
\hline Humility & $.914 * * *$ & $.704^{* * *}$ \\
\hline Universalism-nature & $.815^{* * *}$ & $.584 * *$ \\
\hline Universalism-concern & $.955 * * *$ & $.649 * * *$ \\
\hline Universalism-tolerance & $.884 * * *$ & $.880 * * \star$ \\
\hline Benevolence caring & $.622 * * *$ & $.696 * * *$ \\
\hline $\begin{array}{l}\text { Benevolence } \\
\text { dependability }\end{array}$ & $.528^{\star}$ & $.742 * * *$ \\
\hline
\end{tabular}

$\star * * p<.001$

${ }^{* *} p<.01$.

${ }^{\star} p<.05$, all one tailed.

correlation in each row and column of the valuebehavior correlation matrix should be on the diagonal, and correlations should decrease as one moves away from the diagonal in each row and column. The pattern of correlations starting from the diagonal would then resemble a $U$ shape. We tested hypothesis 5 by correlating the value-behavior correlations in the columns and rows of the matrix in Table 5 with the polynomial coefficients of a quadratic, U-shaped, trend (Bardi \& Schwartz, 2003; Strang, 2006). ${ }^{7}$ We did this for each value across the 19 behaviors and for each behavior across the 19 values. The resulting correlations indicate how well the patterns of value-behavior correlations approximate a sinusoid curve.

Column 1 of Table 7 lists the approximation correlations with a sinusoid curve for each value across all behaviors. Column 2 lists the correlations for each behavior across all values. For values, the mean correlation was .83, for behaviors .80 (both $p<.001$, two tailed, with $r$ to $z$ transformation). The approximation correlations supported the hypothesis for all 19 values and 19 behaviors (both $p<.05$, two tailed, with $r$ to $z$ transformation). In separate analyses for each sample (Supplement E), mean correlations for countries ranged from .72 (Poland) to .84 (Italy) for

\footnotetext{
${ }^{7}$ We used the following coefficients, starting from the correlation between each value and its own behavior: $10,9,8,7,6,5,4,3,2,1,1$, $2,3,4,5,6,7,8,9$. We specified only one highest coefficient because we expected only one peak and two smallest coefficients because, with an odd number of categories (19), each category has two maximally opposed categories.
}

values and from .65 (Russia) to .81 (Italy) for behaviors. The mean correlations across countries were .76 for values and .72 for behaviors. Thus, relations of many different values with many different behaviors reflect the same motivational conflicts and compatibilities captured by the circular continuum of values.

\section{Moderators of the Strength of the Value-behavior Association}

Normative pressure. Bardi and Schwartz (2003) found that value-behavior associations are weaker for behaviors that group members frequently perform and for values that the group endorses highly. We tested this claim in our four countries by correlating the order of the 19 value-behavior correlations in each country with the order of the mean importance of the 19 values and of the mean frequency of the 19 behaviors. The assumption that normative pressure moderates value-behavior relations implies that the correlations should be negative. Table 8 presents the Spearman correlations obtained in each country. The correlation with value importance was significantly negative in Italy, significantly positive in Russia, and not significant in Poland and the USA. The correlation with behavior frequency was significantly negative in Italy and not significant in the other countries.

Gender. Schwartz and Butenko (2014) suggested that gender might moderate the strength of valuebehavior relations. They expected stronger valuebehavior relations for power among women and for benevolence among men, as explained in the introduction. We examined possible moderation by gender by regressing each of the 19 behaviors on its corresponding value, on gender, and on the multiplicative interaction between the value and gender. We standardized gender and the value factor score before forming the interaction term. Supplement $\mathrm{F}$ reports results of the regression analyses in each country. Our analyses did not replicate the finding of stronger relations for power among women in any of the four samples. For benevolence-caring, we found stronger relations among men in Poland but among women in Italy. For benevolence-dependability, we also found stronger relations among men in Poland. For humility, relations were stronger among men in Italy but among women in Russia. Gender moderated the strength of value-behavior relations $(p<.05)$ in only seven of 76 ( 4 country $\times 19$ values) analyses.

\section{Discussion}

This research sought to assess whether each of the 19 values in the refined theory predicts behavior distinctively. It examined relations of values to behavior, measured by combined self-reports and other-reports, in four socioeconomically and culturally diverse countries. Correlations between self-rated and other-rated behaviors were substantial, averaging .43 . Analyses of responses to the PVQ-R, using MGCFA 
Table 8. Spearman rank correlations of the strength of value-behavior relations with value importance and behavior frequency

\begin{tabular}{lcccc}
\hline Correlations of the strength of value-behavior relations with: & Poland & Italy & USA & Russia \\
\hline Value importance & .018 & $-.568^{* *}$ & -.251 & -.082 \\
Behavior frequency ratings & -.164 & $-.544^{* *}$ & -.174 & .068 \\
\hline
\end{tabular}

${ }^{* *} p<.01$.

${ }^{*} p<.05$, one tailed.

and MDS, established that respondents in each country discriminated all 19 values and that the values were arrayed on the theorized circular motivational continuum. This made it possible to test the hypothesized relations of values to behavior. Separate analyses of value-behavior relations for the self-rated and other-rated behaviors showed a similar pattern. Four types of analyses supported the predictive and discriminant validity of the 19 values.

First, in the combined sample correlation matrix, all 19 correlations of a value with the behavior for which it was expected to be the primary motivator were positive and significant. Moreover, in the matrices of all four countries, all 19 values correlated positively with the behavior for which they were expected to be the primary motivator and $97 \%$ were significant. These findings support Hla.

Second, in the combined sample correlation matrix, correlations of values with their corresponding behavior exceeded correlations with other behaviors in $98 \%$ of comparisons. Moreover, for all but achievement values, no other value correlated significantly more strongly with their corresponding behavior. Across the matrices of the four countries, correlations of values with their corresponding behavior exceeded their correlations with other behaviors in $95 \%$ of comparisons. These findings support hypothesis $1 \mathrm{~b}$.

Third, in the combined sample correlation matrix, each behavior correlated more positively with its corresponding value than with any other value in $98 \%$ of comparisons. This was also the case in $96 \%$ of comparisons across the matrices of the four countries. The correlation differences were significant in $97 \%$ of comparisons in the combined matrix and in $94 \%$ across countries. These findings support hypotheses $2 \mathrm{a}$ and $2 \mathrm{~b}$.

Fourth, in the combined sample matrix, values correlated negatively with behaviors presumably motivated by an opposing higher-order value in $95 \%$ of cases across countries. The only exceptions were two nearzero correlations of achievement values and of face values with benevolence behaviors. Across the matrices of the four countries, the hypothesized negative correlations of values emerged in $93 \%$ of comparisons. These findings support hypothesis 3.

\section{Value Tradeoffs}

This study provided the first systematic test of the key idea of the value theory inherent in the notion of the motivational circle: behavior is a product of tradeoffs between values that propel it and values that oppose it. Regressions of each behavior on its corresponding value and opposing higher-order value addressed this idea. In 75 of the 76 regressions, values predicted their corresponding behavior, 71 significantly. For the opposing higher-order values, 72 of the betas were negative, but only 41 were significant. The regressions provide some support for the tradeoff hypothesis (hypothesis 4). In the HLM summary analysis across countries, all corresponding values predicted significantly, and all but one of the betas for opposing values were negative. But only nine were significant. Thus, the values expected to propel behavior related more strongly and consistently to behavior than did the values expected to inhibit it.

Might this stronger association of propelling versus inhibiting values be a general characteristic of valuebehavior relations? In the current study, inhibiting values did not have an equal chance. First, each of the 19 sets of behavior was chosen with the expectation that a particular value would motivate it positively, without regard for the values that might inhibit it. Second, we operationalized the expected inhibiting values as the higher-order value opposed to the propelling value in the motivational circle. It is likely that some component values of each higher-order value were less relevant to the behavior than others were, thereby weakening the observed associations. In order to assess the relative importance of propelling and inhibiting values in value tradeoffs, it is necessary to find behaviors that are specifically and equally relevant to both values.

The slopes of the behaviors on the values that propelled and opposed them varied across the four countries in 23 of the 38 cases. Variation across countries was neither greater for the values that propelled behavior compared with those that opposed behaviors nor for values from any one of the four higher-order values (all $p>.15$ by $\chi^{2}$ test). However, the most frequent sources of significant variation were the slopes in the USA (10 cases) and Russia (9 cases). In almost every case, the effect in these two countries was the weakest. The fact that respondents in the USA and Russia knew each other for considerably fewer years than respondents in Poland and Italy (mean 6.5 and 2.5 vs. 10.6 and 14.6, respectively, $F$ (3, $1958)=84.85, p<.001)$ may explain this. In the USA and Russia, other ratings were substantially poorer predictors of behavior than self ratings, whereas the two ratings differed much less in Poland and Italy.

\section{Overlapping Content: An Alternative Explanation for Value-behavior Relations}

Might the content of the behaviors themselves explain the structure of their relations to values? This does not 
seem likely (cf. Schwartz \& Butenko, 2014). There need be no inherent conflict between behaviors that express opposing values. For example, "avoid walking alone on a dark street at night" (security-personal) need not conflict with "change plans spontaneously" (stimulation). These behaviors are opposed because security values motivate one and stimulation values motivate the other. Furthermore, there need be no inherent similarity between behaviors that express compatible values. "Insist that others do what I want" (power-dominance) and "buy luxury brands of clothing so that other people will notice" (power-resources) are not inherently similar behaviors. What makes them compatible is that both express a motivation for power and control, whether over people or resources. In sum, the motivational conflicts and compatibilities that structure relations among values largely guide their relations with behaviors.

\section{Moderators of Value-behavior Relations}

Normative pressure. Bardi and Schwartz (2003) found that value-behavior associations are weaker for behaviors that group members frequently perform and for values that the group endorses highly. They suggested that such behaviors and values are normative, so individuals comply with normative expectations rather than behaving according to their own values. The normative pressure explanation for variation in the strength of value-behavior relations is often cited and widely accepted (e.g., Fischer et al., 2009; Hitlin \& Piliavin, 2004; Lipponen et al., 2008; Lönnqvist et al., 2006). It did not replicate in the Schwartz and Butenko (2014) Russian study with self-reported behavior, however.

The strength of value-behavior relations correlated negatively with value importance in Italy but positively in Russia in the current study, and the correlations were not significant in Poland and the USA. The strength of value-behavior relations also exhibited inconsistent correlations with behavior frequencies, negative in Italy but not significant in the other in three countries. In sum, studies in two countries, Israel and Italy, have supported the normative pressure explanation, whereas studies in three countries have not. This challenges researchers to identify conditions under which normative pressure does or does not undermine value-behavior relations. The opposing findings for Italy and Russia in the same study make it unlikely that differences in study design produced the inconsistency.

An alternative approach could draw on the idea of cultural differences in the extent to which internal attributes predict behavior (Church, 2008). A possible explanation might draw on the tightness-looseness of the national culture. Tighter cultures have stronger social norms and less tolerance for deviance (Gelfand et al., 2011; Uz, 2015). In tighter cultures, we might expect individuals to be more sensitive to and compliant with normative pressures rather than acting on their own values. Scores for tightness are available for the five countries mentioned here in Gelfand et al. (2011; except Russia) and Uz (2015; except Israel). Contrary to expectations, one of the countries in which the normative pressure explanation was supported (Israel) scored lowest on tightness and the other (Italy) scored lowest on tightness in Uz (2015) but highest in Gelfand et al. (2011). Thus, the challenge remains to identify the dimension(s) that distinguish Israel and Italy from Poland, Russia, and the USA that might account for the different observed effects of normative pressure on value-behavior relations.

Gender. Schwartz and Butenko (2014) suggested that gender might moderate the strength of value-behavior relations. They expected stronger value-behavior relations for power among women and for benevolence among men. They had no expectations for gender differences for other value domains. Their findings confirmed the moderating role of gender for power values but not for benevolence values. They also obtained several unexpected, significant interactions. Our analyses did not replicate the finding of stronger relations for power among women in any of the four samples. For benevolence-dependability and benevolence-caring, we found stronger relations among men than among women only in Poland. Gender moderated the strength of value-behavior relations $(p<.05)$ in only seven of 76 ( 4 country $\times 19$ values) analyses. This could easily be a chance result. Hence, systematic gender differences in the strength of value-behavior relations seem unlikely.

\section{Order of the Values in the Motivational Circle}

As in the MDS projections in the 15 Schwartz et al. (2012) samples, the two benevolence values were adjacent to the openness to change higher-order values and the three universalism values were adjacent to the higher-order conservation values in our four samples. They used centered raw scores for 48 value items in the MDS analyses, whereas we used factor scores for 19 values. Our findings, based on somewhat different measures, reinforce the conclusion that this is the appropriate order of the values in the refined theory, rather than the order that Schwartz et al. (2012) initially hypothesized. This study also provided a new type of evidence for the circular motivational continuum of the value theory. The patterns of correlation between each value and all 19 behaviors and between each behavior and the 19 values, assessed by the sinusoid curves, largely reproduced the circular order of the theory. Thus, not only did relations among values reveal the motivational circle but relations of values to behavior did so too.

The MDS projections also confirm that the PVQ-R can be used to form indices of the original 10 values or the four higher-order values. ${ }^{8}$ The refined theory discriminated facets of conformity, security, power,

\footnotetext{
${ }^{8}$ We recommend using the improved and revised PVQ-RR, which is available from the first author, to measure the values.
} 
self-direction, universalism, and benevolence values. In the MDS analyses, the facets of each of these original values were adjacent (e.g., power-dominance adjacent to power-resources). Moreover, the values that constitute each higher-order value formed a unique region.

\section{Future Directions}

Causal relations between values and behavior. The current study supported the validity of the values through their links with behavior. It did not test the implicit assumption that at least some of that association is causal, linking values to behavior through both motivational and cognitive processes. As expressions of underlying motivations in the form of goals, values make behavior that promotes these goals more attractive and motivate such behavior (Feather, 1995; Roccas \& Sagiv, 2010; Rokeach, 1973). As mental representations of desirable abstract goals, values promote behaviors that are cognitively associated with and instantiate these goals (Maio, 2015). Longitudinal studies are needed to assess the causal role of the 19 values in predicting behavior. Experimental studies that manipulate the salience of the values and examine the impact on behavior and on value-behavior relations are also desirable. Past longitudinal and experimental studies of this sort with the original 10 values can serve as models (e.g., Maio et al., 2009; Schwartz, Caprara, \& Vecchione, 2010). Such studies are usually limited to investigating only one or two values at a time, but a systematic series of studies could assess all 19 values.

Values and the study of morality. Like other theories of values, morality, and culture (e.g., Graham et al., 2012; Shweder, Much, Mahapatra, \& Park, 1997), ours seeks to identify basic principles that are recognized as desirable by individuals across cultures and that may guide cognition, emotion, and behavior. Basic values, like moral foundations, typically function as automatic intuitions whose influence occurs outside of awareness; reasoning about the value bases of judgments and actions comes later and often serves to justify them (Schwartz, 2006). Basic values are more fine-tuned than the foundations of morality.

If lists of the foundations of morality are reasonably complete, each of the 19 basic values should represent a virtue relevant to at least one foundation. The currently dominant theory specifies five foundations (Graham et al., 2012). Most values clearly constitute virtues relevant to one or another foundation. For example, we can link benevolence caring to Care, universalism-tolerance to Fairness, and tradition to Loyalty, Authority, and Sanctity. Might basic values as narrower expressions of the moral foundations mediate their relations to attitudes and behavior? Few studies have examined moral foundations and basic values together (e.g., Haidt, Graham, \& Joseph, 2009). None has addressed this plausible hypothesis.

Matching the values to the five foundations suggests many questions both about the values and the moral foundations. We mention six that might stimulate further thinking. Self-direction values are among the most important values across cultures (Schwartz \& Bardi, 2001). Yet, none of the five moral foundations clearly underlies them. The wide-spread importance of these values supports accepting the proposed Liberty as a sixth foundation. We also find no match for achievement values. This supports the proposed Industry foundation that it would match. Conformity-rules represent the group-oriented moral foundations of Loyalty, Authority, and Sanctity, associated with conservative ideologies. In contrast, conformity-interpersonal represents the individual-oriented moral foundations of Care and Fairness, associated with liberal ideologies. Yet, the two types of conformity values are closely related in value analyses. Might this suggest that the two types of conformity values are more distinct than the value theory recognizes or that the distinction between the two broad sets of moral foundations is over-stated? Benevolence caring is a virtue relevant to the foundation of Care, and benevolence dependability is a virtue more linked to the foundation of Loyalty. Are the two types of benevolence more distinct than the value theory assumes or are the two foundations less distinct? Relations among the moral foundations have received little consideration. Are they orthogonal or, like values, might they form some sort of continuum?

Choice and measurement of behaviors. Our goal of validating the values by relating them to behavior led to a priori selection of behaviors that we expected to express each value. Researchers might focus, instead, on behaviors chosen because they are of particular interest in themselves. It would be interesting to examine the extent to which the 19 values explain these "neutral" behaviors that are likely to express multiple values. Such studies could profitably include a scale to measure basic traits (e.g., the Big Five). This would permit assessing the relative contributions to explaining such behaviors of values, traits, and their interactions (cf. Caprara, Schwartz, Capanna, Vecchione, \& Barbaranelli, 2006). Studies of varied behaviors could clarify the types of behavior that values versus traits explain more effectively. Past research provides limited data indicating that values predict deliberate behavior better and traits predict affective, automatic responses better (e.g., Roccas et al., 2002). Further research could evaluate this view and try to identify other characteristics of behavior that influence the relative predictive power of values and traits.

It is important to go beyond reports of behavior to investigate how the 19 values relate to actual, objectively measured behavior (Baumeister, Vohs, \& Funder, 2007). This is difficult to do in any single study for 19 different types of behavior, of course. One method that may provide behavioral data relevant to several values and that comes closer to actual behavior is experience sampling in real time over several weeks. 


\section{Conclusions}

The refined value theory (Schwartz et al., 2012) proposed that partitioning the circular motivational continuum into a finer set of meaningful values could yield increased heuristic and predictive power. Past research (Schwartz et al., 2012) supported the discriminant and predictive validity of the more narrowly defined values of the theory by examining their associations with attitude, belief, and background variables. A Russian study, using self-reports, found some evidence for relations of the values to behavior (Schwartz and Butenko, 2014). The current study, which measured behavior with combined self-reports and other-reports in four countries, provided a convincing validation of the values by relating them to behavior.

Although we measured reported rather than actual behavior, combining other-reports with self-reports improved the behavior indices. Self-reports draw on targets' knowledge of their own behavior that is unavailable to others; other-reports are less vulnerable to selfconsistency and defensive biases. Self-other agreement was high enough to suggest that targets and others reported on some of the same actual behavior, but low enough to suggest that each report added important information. The fact that self-other agreement on values is as high as self-other agreement on personality traits and on act frequencies is interesting in itself.

In keeping with the theory, values related negatively to the behaviors they were expected to inhibit, not only positively to the behaviors they were expected to promote. This accords with the basic assumption of the theory that values influence both what people do and what they do not do. The regression analyses in this study were the first to reveal the effects of value tradeoffs. The HLM results, summarizing all four samples, provided the first available estimates of the variance in behavior that value tradeoffs explain. Across the 19 domains, the value expected to propel a behavior and the higher-order value expected to oppose it explained an average of over $16 \%$ of the variance. Considering the large number of influences on any behavior, this strengthens the view that values make a meaningful contribution to understanding behavior. Moreover, the current evidence for value tradeoffs suggests that future studies of the relations of behavior to values, in contrast to the vast majority of past research, should include not only the values expected to propel a behavior but also those expected to oppose it.

\section{Acknowledgements}

We are grateful to Anna Czyżkowska and to Anna Lipatova for the help in gathering the data. The work of the first author on this paper was partly supported within the framework of the Basic Research Program at the National Research University Higher School of Economics (HSE) and supported within the framework of a subsidy by the Russian Academic Excellence Project '5-100'. The work of the second author was supported by Grants 2014/14/M/ HS6/00919 from the National Science Centre, Poland.

\section{Supporting information}

Additional supporting information may be found in the online version of this article at the publisher's web-site.

\section{References}

Bardi, A., \& Schwartz, S. H. (2003). Values and behavior: Strength and structure of relations. Personality and Social Psychology Bulletin, 29, 1207-1220. http://dx.doi.org/ 10.1177/0146167203254602

Baumeister, R. F., Vohs, K. D., \& Funder, D. C. (2007). Psychology as the science of self-reports and finger movements: Whatever happened to actual behavior? Perspectives on Psychological Science, 2, 396-403. http://dx. doi.org/10.1111/j.1745-6916.2007.00051.x

Best, D. L., \& Thomas, J. J. (2004). Cultural diversity and cross-cultural perspectives. In A. H. Eagley, A. E. Beall, $\delta$ R. J. Sternberg (Eds.), The psychology of gender $\left(2^{\text {nd }}\right.$ ed., pp. 296-327). New York: Guilford Press.

Bilsky, W., Janik, M., \& Schwartz, S. H. (2011). The structural organization of human values: Evidence from three rounds of the European Social Survey (ESS). Journal of Cross-Cultural Psychology, 42, 759-776. http://dx. doi.org/10.1177/0022022110362757

Borg, I., Groenen, P. J. F., \& Mair, P. (2013). Applied multidimensional scaling. New York: Springer.

Buss, D. M., \& Craik, K. H. (1983). The act frequency approach to personality. Psychological Review, 90, 105-126. http://dx.doi.org/10.1177/0146167289151007

Byrne, B. M., Shavelson, R. J., \& Muthen, B. (1989). Testing for the equivalence of factor covariance and mean structures-the issue of partial measurement invariance. Psychological Bulletin, 105, 456-466. http://dx.doi.org/ 10.1037/0033-2909.105.3.456

Caprara, G. V., Schwartz, S. H., Capanna, C., Vecchione, M., \& Barbaranelli, C. (2006). Personality and politics: Values, traits, and political choice. Political Psychology, 27, 1-28. http://dx.doi.org/10.1111/j.1467-9221.2006.00447.x

Chen, F. (2007). Sensitivity of goodness of fit indices to lack of measurement invariance. Structural Equation Modeling, 14, 464-504.

Church, A. T. (2008). Current controversies in the study of personality across cultures. Social and Personality Psychology Compass, 2, 1930-1951. http://dx.doi.org/10.1111/j.17519004.2008.00132.x

Cieciuch, J., \& Schwartz, S. H. (2012). The number of distinct basic values and their structure assessed by PVQ-40. Journal of Personality Assessment, 94, 321-328. http://dx.doi.org/10.1080/00223891.2012.655817

Commandeur, J. J. F. (1991). Matching configurations. Leiden, The Netherlands: DSWO Press.

Davidov, E., Meuleman, B., Cieciuch, J., Schmidt, P., Billiet, J. (2014). Measurement equivalence in crossnational research. Annual Review of Sociology, 40, 55-75. http://dx.doi.org/10.1146/annurev-soc-071913-043137

Davidov, E., Schmidt, P., \& Schwartz, S. H. (2008). Bringing values back in: The adequacy of the European Social Survey to measure values in 20 countries. Public Opinion Quarterly, 72, 420-445. http://dx.doi.org/10.1093/poq/nfn035

Doran, C. J. (2009). The role of personal values in fair trade consumption. Journal of Business Ethics 84, 549-563. http://dx.doi.org/10.1007/s10551-008-9724-1 
Eagley, A. H., Wood, W., \& Johannesen-Schmidt, M. C. (2004). Social role theory of sex differences and similarities: Implications for the partner preferences of women and men. In A. H. Eagley, A. E. Beall, \& R. J. Sternberg (Eds.), The psychology of gender (2 ${ }^{\text {nd }}$ ed., pp. 269-295). New York: Guilford Press.

Eliason, B. C., \& Schubot, D. B. (1995). Personal values of exemplary family physicians: Implications for professional satisfaction in family medicine. Journal of Family Practice, 41, 251-256.

Feather, N. (1995). Values, valences, and choice: The influence of values on the perceived attractiveness and choice of alternatives. Journal of Personality and Social Psychology, 68, 1135-1151. http://dx.doi.org/10.1037/ 0022-3514.68.6.1135

Fischer, R., Ferreira, M. C., Assmar, E., Redford, P., Glazer, S., Bor-Shiuan, C., ... Achoui, M. (2009). Individualismcollectivism as descriptive norms: Development of a subjective norm approach to culture measurement. Journal of Cross-Cultural Psychology, 40, 187-213. http://dx.doi.org/10.1177/0022022109332738

Gardikiotis, A., \& Blatzis, A. (2012). 'Rock music for myself and justice to the world!': Musical identity, values, and music preferences. Psychology of Music, 40, 143-163. http://dx.doi.org/10.1177/0305735610386836

Gelfand, M. J., Raver, J. L., Nishii, L., Leslie, L. M., Lun, J., Lim, B. C., ... Yamaguchi, S. (2011). Differences between tight and loose cultures: A 33-nation study. Science, 332, 1100-1 104. http://dx.doi.org/10.1126/science.1197754

Goodwin, R., Realo, A., Kwiatkowska, A., Kozlova, A., Nguyen, L. L. A.. and Nizharadze, G. (2002). Values and sexual behaviour in Central and Eastern Europe. Journal of Health Psychology, 7, 45-56. http://dx.doi.org/ 10.1177/1359105302007001651

Gosling, S. D., John, O. P., Craik, K. H., \& Robins, R. W. (1998). Do people know how they behave? Selfreported act frequencies compared with on-line codings by observers. Journal of Personality and Social Psychology, 74, 1337-1349. http://dx.doi.org/10.1037/0022-3514.74.5.1337 Graham, J., Haidt, J., Koleva, S., Motyl, M., Iyer, R., Wojcik, S. P., \& Ditto, P. H. (2012). Moral foundations theory: The pragmatic validity of moral pluralism. Advances in Experimental Social Psychology, 47, 55-130. http://dx.doi. org/10.1016/B978-0-12-407236-7.00002-4

Haidt, J., Graham, J., \& Joseph, C. (2009). Above and below left-right: Ideological narratives and moral foundations. Psychological Inquiry, 20, 110-119. http://dx.doi.org/ $10.1080 / 10478400903028573$

Harrington, D. (2008). Confirmatory factor analysis. New York: Oxford University Press.

Hartman, J. B., Shim, S., Barber, B., \& O'Brien, M. (2006). Adolescents' utilitarian and hedonic web-consumption behavior: Hierarchical influence of personal values and innovativeness. Psychology $\theta$ Marketing, 23, 813-839. http://dx.doi.org/10.1002/mar.20135

Hitlin, S., \& Piliavin, J. A. (2004). Values: Reviving a dormant concept. Annual Review of Sociology, 30, 359-393. http://dx.doi.org/10.1146/annurev.soc.30.012703.110640

Hu, L., \& Bentler, P. M. (1999). Cut-off criteria for fit indexes in covariance structure analysis: Conventional criteria versus new alternatives. Structural Equation Modeling, 6, 1-55. http://dx.doi.org/10.1080/1070551 9909540118
John, O. P., \& Robins, R. W. (1993). Determinants of interjudge agreement on personality traits: The Big Five domains, observability, evaluativeness, and the unique perspective of the self. Journal of Personality, 61, 521-551. http://dx.doi.org/10.1111/j.1467-6494.1993.tb00781.x

Kasof, J., Chen, C., Himsel, A., \& Greenberger, E. (2007). Values and creativity. Creativity Research Journal, 19, 105-122. http://dx.doi.org/10.1080/10400410701397164

Knafo, A., Daniel, E., \& Khoury-Kassabri, M. (2008). Values as protective factors against violent behavior in Jewish and Arab high schools in Israel. Child Development, 79, 652-667. http://dx.doi.org/10.1111/ j.1467-8624.2008.01149.x

Lipponen, J., Bardi, A., Haapamäki, J. (2008). The interaction between values and organizational identification in predicting suggestion-making at work. Journal of Occupational and Organizational Psychology, 81, 241-248. http://dx.doi.org/10.1348/096317907X216658

Lönnqvist, J.-E.., Leikas, S., Paunonen, S., Nissinen, V., \& Verkasalo, M.. (2006). Conformism moderates the relations between values, anticipated regret, and behavior. Personality and Social Psychology Bulletin, 32, 1469-1481. http://dx.doi.org/10.1177/0146167206291672

Lönnqvist, J.-E., Walkowitz, G., Wichardt, P., Lindeman, M. Verkasalo, M. (2009). The moderating effect of conformism values on the relations between other personal values, social norms, moral obligation, and single altruistic behaviours. British Journal of Social Psychology, 48, 525-546. http://dx.doi.org/10.1348/0144 $66608 \times 377396$

Maio, G. R. (2015). The psychology of human values. European Monographs in Social Psychology. London: Psychology Press.

Maio, G. R., Pakizeh, A., Cheung, W., \& Rees, K. J. (2009). Changing, priming, and acting on values: Effects via motivational relations in a circular model. Journal of Personality and Social Psychology, 97, 699-715. http://dx.doi.org/ $10.1037 / \mathrm{a} 0016420$

Marsh, H. W., Hau, K. T., \& Wen, Z. (2004). In search of golden rules: Comment on hypothesis-testing approaches to setting cut-off values for fit indices and dangers in overgeneralizing $\mathrm{Hu}$ and Bentler's (1999) findings. Structural Equation Modeling, 11, 320-341. http://dx.doi. org/10.1207/s15328007sem1103_2

McCrae, R. R., \& Costa, P. T. Jr. (1987). Validation of the fivefactor model of personality across instruments and observers. Journal of Personality and Social Psychology, 52, 81-90. http://dx.doi.org/10.1037/0022-3514.52.1.81

Mõttus, R., Allik, J., Realo, A., Rossier, J., Zecca, G., Ah-Kion, J., ... Tseung, C. N. (2012). The effect of response style on self-reported conscientiousness across 20 countries. Personality and Social Psychology Bulletin, 38, 1423-1436. http://dx.doi.org/10.1177/0146167212451275

Mount, M. K., Barrick, M. R., \& Strauss, J. P. (1994). Validity of observer ratings of the big five personality factors. Journal of Applied Psychology, 79, 272-280. http://dx.doi. org/10.1037/0021-9010.79.2.272

Muthén, L., \& Muthén, B. O. (1998-2012). Mplus User's Guide (7th ed.). Los Angeles, CA: Muthén and Muthén.

Paulhus, D. L., \& Vazire, S. (2009). The self-report method. In R. W. Robins, R. C. Fraley, \& R. F. Krueger (Eds.), Handbook of research methods in personality psychology (pp. 224-249). New York: Guilford. 
Raudenbush, S. W., \& Bryk, A. S. (2002). Hierarchical linear models: Applications and data analysis methods. Newbury Park, CA: Sage.

Roccas, S., \& Sagiv, L. (2010). Personal values and behavior: Taking the cultural context into account. Social and Personality Compass, 4, 31-41. http://dx.doi.org/10.1111/ j.1751-9004.2009.00234.x

Roccas, S., Sagiv, L., Schwartz, S. H., \& Knafo, A. (2002). The Big Five personality factors and personal values. Personality and Social Psychology Bulletin, 28, 789-801. http://dx.doi.org/10.1177/0146167202289008

Rokeach, M. (1973). The nature of human values. New York: Free Press.

Sagiv, L., Sverdlik, N., \& Schwarz, N. (2011). To compete or to cooperate? Values' impact on perception and action in social dilemma games. European Journal of Social Psychology, 41, 64-77. http://dx.doi.org/10.1002/ejsp.729

Saris, W. E., Knoppen, D., \& Schwartz, S. H. (2013). Operationalizing the theory of human values: Balancing homogeneity of reflective items and theoretical coverage. Survey Research Methods, 7, 29-44.

Schwartz, S. H. (1992). Universals in the content and structure of values: Theory and empirical tests in 20 countries. In M. Zanna (Ed.), Advances in experimental social psychology (Vol. 25) (pp. 1-65). New York: Academic Press. http://dx.doi.org/10.1016/S0065260108602816

Schwartz, S. H. (1994). Are there universal aspects in the content and structure of values? Journal of Social Issues, 50, 19-45. http://dx.doi.org/10.1111/j.1540-4560.1994. tb01196.x

Schwartz, S. H. (2006). Les valeurs de base de la personne: Théorie, mesures et applications [Basic human values: Theory, measurement, and applications]. Revue Française de Sociologie, 47, 249-288.

Schwartz, S. H. (2010). Basic values: How they motivate and inhibit prosocial behavior. In M. Mikulincer, \& P. Shaver (Eds.), Prosocial motives, emotions, and behavior: The better angels of our nature (pp. 221-241). Washington: American Psychological Association.

Schwartz, S. H. (2015). Basic individual values: Sources and consequences. In D. Sander and T. Brosch (Eds.), Handbook of value (pp. 63-84). Oxford, UK: Oxford University Press.

Schwartz, S. H., \& Bardi, A. (2001). Value hierarchies across cultures: Taking a similarities perspective. Journal of Cross-Cultural Psychology, 32, 268-290. http://dx.doi.org/ $10.1177 / 0022022101032003002$

Schwartz, S. H., \& Butenko, T. (2014). Values and behavior: Validating the refined value theory in Russia. European Journal of Social Psychology, 44, 719-813. http://dx.doi.org/ 10.1002/ejsp.2053

Schwartz, S. H., Caprara, G. V., \& Vecchione, M. (2010). Basic personal values, core political values, and voting: A longitudinal study. Political Psychology, 31, 421-452. http:// dx.doi.org/10.1111/j.1467-9221.2010.00764.x

Schwartz, S. H., Cieciuch, J., Vecchione, M., Davidov, E., Fischer, R., Beierlein, C., ... Konty, M. (2012). Refining the theory of basic individual values. Journal of Personality and Social Psychology, 103, 663-688. http://dx.doi.org/ 10.1037/a0029393

Schwartz, S. H., \& Rubel, T. (2005). Sex differences in value priorities: Cross-cultural and multi-method studies. Journal of Personality and Social Psychology, 89, 1010-1028. http://dx.doi.org/10.1037/0022-3514.89.6.1010

Schultz, P. W., \& Zelezny, L. C. (1998). Values and proenvironmental behavior: A five-country survey. Journal of Cross-Cultural Psychology, 29, 540-558. http://dx.doi. org/10.1177/0022022198294003

Shweder, R. A., Much, N. C., Mahapatra, M., \& Park, L. (1997). The "big three" of morality (autonomy, community, and divinity), and the "big three" explanations of suffering. In A. Brandt, \& P. Rozin (Eds.), Morality and health (pp. 119-169). New York: Routledge.

Steiger, J. H. (1980). Tests for comparing elements of a correlation matrix. Psychological Bulletin, 87, 245-251. Update retrieved from http://www.psychmike.com/ dependent_correlations.php

Strang, G. (2006). Linear algebra and its applications. Belmont, CA: Thomson Brooks/Cole.

Tamayo, A., Nicaretta, M., Ribeiro, R., \& de Barbosa, L. P. G. (1995). Prioridades axiológicas y consumo de drogas. [Value priorities and the use of drugs]. Acta Psiquiatrica $y$ Psicológica de América Latina, 41, 300-307.

Uz, I. (2015). The index of cultural tightness and looseness across 68 countries. Journal of Cross-Cultural Psychology, 46, 319-335. http://dx.doi.org/10.1177/0022022114563611

Vazire, S., \& Mehl, M. R. (2008). Knowing me, knowing you: The accuracy and unique predictive validity of self-ratings and other-ratings of daily behavior. Journal of Personality and Social Psychology, 95, 1202-1216. http://dx.doi.org/ 10.1037/a0013314

Verplanken, B., \& Holland, R. W. (2002). Motivated decision making: Effects of activation and self-centrality of values on choices and behavior. Journal of Personality and Social Psychology, 82, 434-447. http://dx.doi.org/10.1037/00223514.82.3.434

Wagerman, S. A., \& Funder, D. C. (2007). Acquaintance reports of personality and academic achievement: A case for conscientiousness. Journal of Research in Personality, 41, 221-229. http://dx.doi.org/10.1016/j.jrp.2006.03.001

Van de Vijver, F., \& Poortinga, Y. (1994). Methodological issues in cross-cultural studies on parental rearing behavior and psychopathology. In C. Perris, W. A. Arrindell, \& M. Eisemann (Eds.), Parental rearing and psychopathology (pp. 173-197). Chichester: Wiley. 